\title{
Preliminar Propagation and MIMO Experiments in Train Tunnels at $5.8 \mathrm{GHz}$
}

\author{
J. Alonso, B. Izquierdo, S. Capdevila, and J. Romeu \\ AntennaLab, Dept. of Signal Theory and Communications (TSC), \\ Universitat Politècnica de Catalunya (UPC), Barcelona, Spain. \\ E-mail: \{javalde,izquierdo,capdevila,romeu\}@tsc.upc.edu
}

\section{Introduction}

In [1] and [2] a set of tools were presented in order to characterize radiowave propagation in train tunnels at $5.8 \mathrm{GHz}$. An experimental campaign was carried over in a straight tunnel at Barcelona L2 Metro and results were presented and compared with theoretical results. In this paper, results of a new measurement campaign at $5.8 \mathrm{GHz}$ for a different tunnel geometry are presented. A curved tunnel section has been measured for SISO and 2x2 MIMO schemes to provide more accuracy for radiowave propagation characterization in tunnels and quantify the improvement attained when MIMO technologies are used.

\section{Measurements Environment and Experimental Setup}

The experimental campaign was done at Barcelona L9 Metro. The cross-section geometry of L9 differs notoriously from the scenario described in [2]: a 6m-radius circular shape split in two halves that can be observed in Fig.1. Measurements are taken in the lower half of the tunnel, in a right-bended stretch of $300 \mathrm{~m}$ and curvature radius of $288 \mathrm{~m}$.

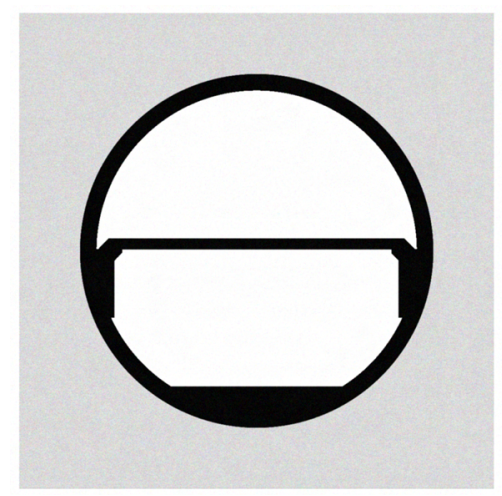

(a) L9 cross-section scheme

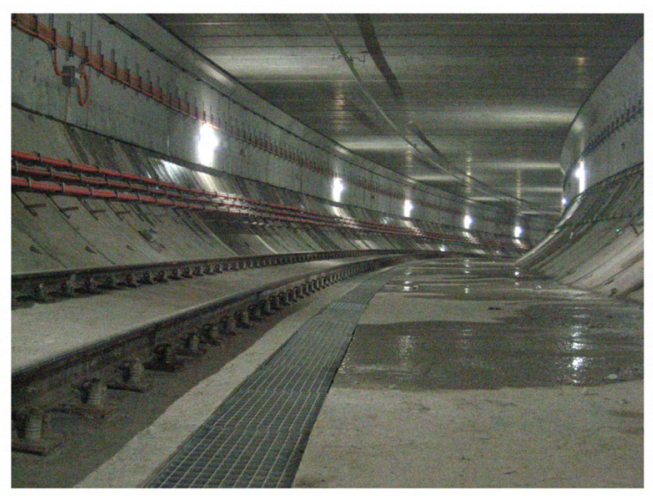

(b) L9 lower section

Figure 1: L9 Tunnel

Setup for the SISO experiment is described in [2]. Tx is placed $2.3 \mathrm{~m}$ left from the tunnel axis at $2 \mathrm{~m}$ height.Rx is aligned with the $\mathrm{Tx}$ at the beginning of each set of measurements (HH,VV and VH polarizations). Fig.2 shows the MIMO setup. Two Software Defined Radio (SDR) laptop-controlled cards are used as Tx and $\mathrm{Rx}$ respectively. Both transmitting and receiving antennas are composed of two resonating square patches in a $1 \mathrm{x} 2$ array configuration, connected to each SDR 
card, centered $1.7 \mathrm{~m}$ left of the tunnel axis, $1.4 \mathrm{~m}$ height. The Tx generates two delta-tones centered around $5.8 \mathrm{GHz}$ frequency-spaced $10 \mathrm{KHz}$. The $\mathrm{Rx}$ is mounted on a sliding platform manually pushed and captures data every $0.25 \mathrm{~m}$. Again, three sets of measurements were captured. Polarization for Tx and Rx is identical for each set: first both antennas of each array operate in $\mathrm{V}$ polarization (VV), afterwards in $\mathrm{H}$ polarization $(\mathrm{HH})$, and finally one antenna operates in $\mathrm{V}$ and the other in $\mathrm{H}$ $(\mathrm{VH})$.

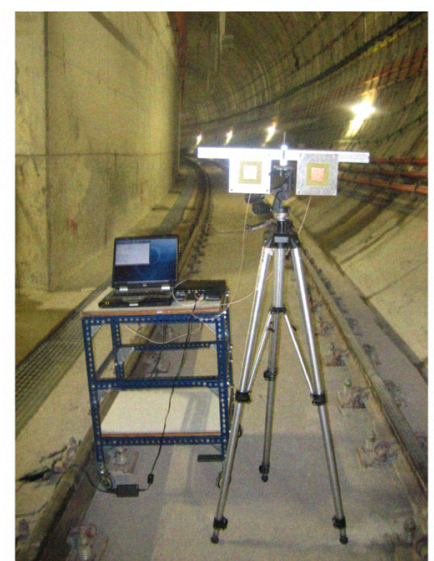

(a) MIMO transmitter

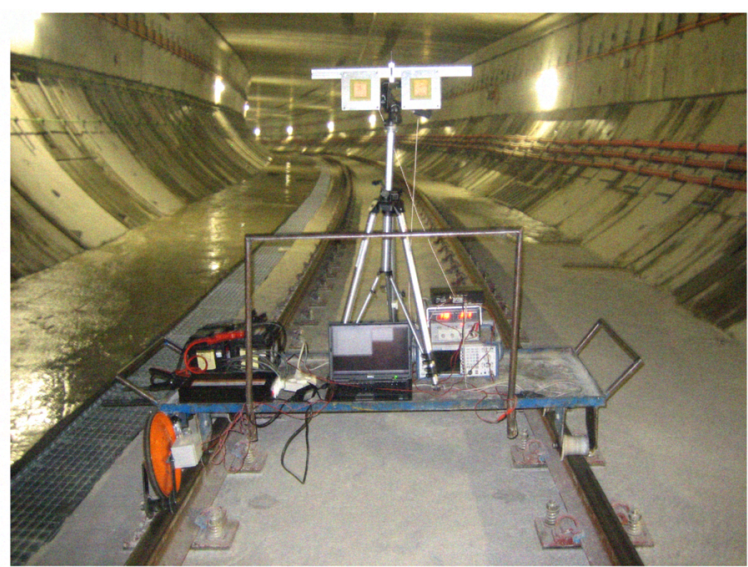

(b) MIMO receiver

Figure 2: MIMO setup

\section{Measurement Results}

In this section the propagation path loss curves and the preliminar MIMO results are presented. The propagation path losses are presented in Fig. 3 where the same behaviour observed at [2] for curved tunnels is present at the curves. These propagation path losses curves allow to analyse the behaviour of the MIMO set-up in terms of received power for different configurations.

The Fig. 4 summarises the singular value behaviour along the bottom section of

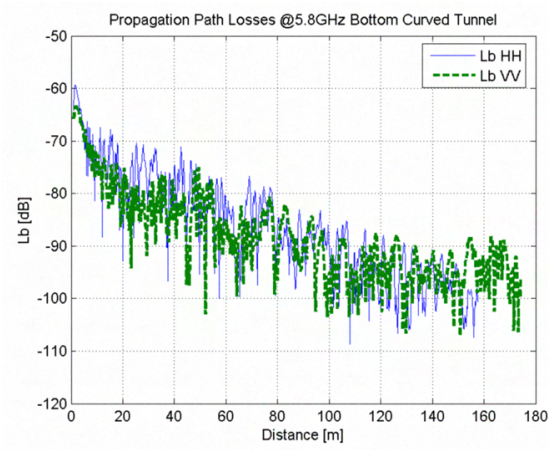

(a) Bottom section of the tunnel propagation path losses

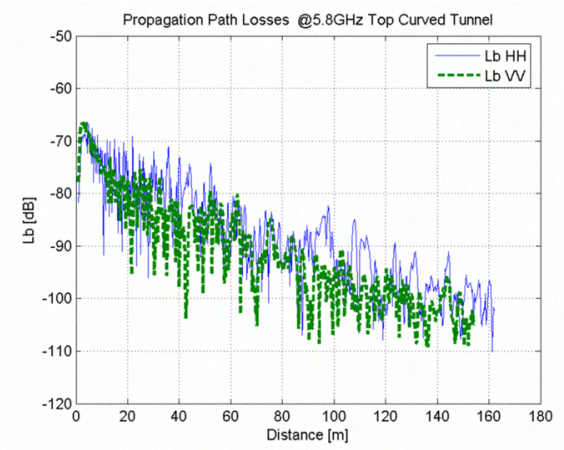

(b) Top section of the tunnel propagation path losses

Figure 3: SISO propagation path losses at $5.8 \mathrm{GHz}$

the curved tunnel showing the singular value relation $\frac{\min \left(\sigma_{i}\right)}{\max \left(\sigma_{i}\right)}$ for different antenna 
spacing $(\lambda, 3 \lambda)$ and different polarisation (VV and $\mathrm{VH})$ which is an example of the expected results [3]. In this curve $\sigma_{i}$ are the set of singular values of the normalised channel matrix. With this representation a ratio of 1 means equal singular values and a full availability of independent channels. On the other hand, a ratio of 0 means the existence of a single channel.

In Fig. 4 erratic behaviour of the singular value relation can be observed at $\mathrm{VV}$ and $\mathrm{VH}$ polarisation with $\lambda$ and $3 \lambda$ antenna spacing. However, the VV configuration with $3 \lambda$ antenna spacing shows a less erratic behaviour in the singular value relation due to a lower influence of the deep fading over this transmitter/receiver configuration. This observation leads to a preliminar conclusion about the MIMO capacity advantages in subway tunnels based on the results presented at [4] where the depolarisation in arched tunnels is studied. Note that analysis has been performed with a equal distributed transmitting power.

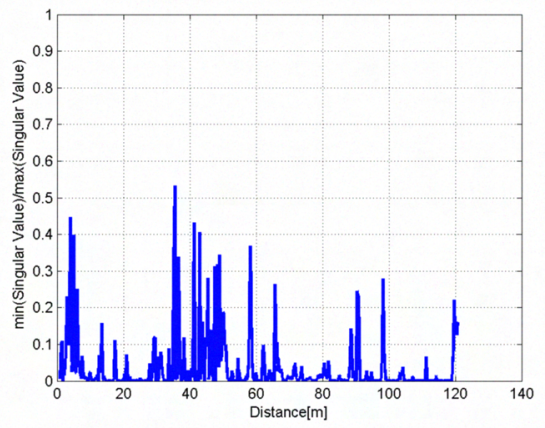

(a) $\frac{\min \left(\sigma_{i}\right)}{\max \left(\sigma_{i}\right)}$ for $\mathrm{VV}$ configuration with $\lambda$ antenna spacing

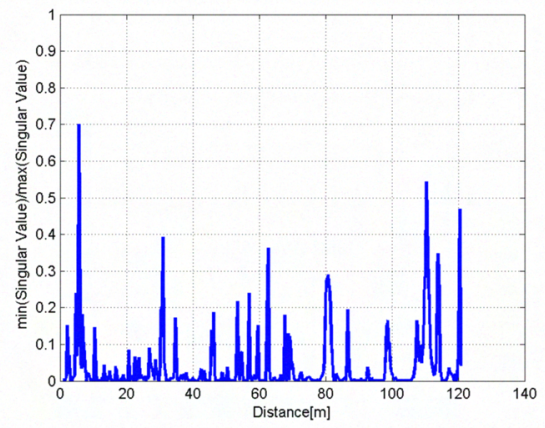

(b) $\frac{\min \left(\sigma_{i}\right)}{\max \left(\sigma_{i}\right)}$ for VV configuration with $3 \lambda$ antenna spacing

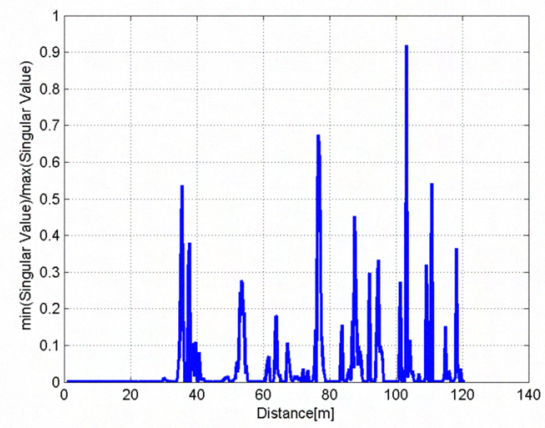

(c) $\frac{\min \left(\sigma_{i}\right)}{\max \left(\sigma_{i}\right)}$ for VH configuration with $3 \lambda$ antenna spacing

Figure 4: $\frac{\min \left(\sigma_{i}\right)}{\max \left(\sigma_{i}\right)}$ for different antennas polarisation and antenna spacing

\section{Conclusions}

At this paper, a complete set-up to subway radio propagation measurement at $\mathrm{L}$ and $\mathrm{C}$ Band is presented. This set-up has been tested at a real subway tunnel with a special structural characteristics allowing propagation path loss measurements and MIMO analysis in order to study the best system configuration working in subway 
environments.

Therefore, the next step is to present a new measurement camping where a complete characterisation of the diversity possibilities inside a straight and curved tunnel will be provided. This new campaign will take place at the L9 Barcelona Metro Network new facilities.

\section{Acknowledgments}

This work has been sponsored by IFERCAT (Infraestructures Ferroviàries de Catalunya) and was supported in part by the Spanish Interministerial Commission on Science and Technology (CICYT) under projects TEC2007-66698-C04-01/TCM

and CONSOLIDER CSD2008-00068 and by the "Ministerio de Educaciòn y Ciencia" through the FPU fellowship program.

\section{References}

[1] B. Izquierdo, S. Capdevila, L. Jofre, and J. Romeu, "Evaluation of MIMO capacity in train tunnels," in Antennas and Propagation International Symposium, 2007 IEEE, S. Capdevila, Ed., 2007, pp. 1365-1368.

[2] J. Alonso, S. Capdevila, B. Izquierdo, and J. Romeu, "Propagation measurements and simulations in tunnel environment at 5.8ghz," in Antennas and Propagation Society International Symposium, 2008. AP-S 2008. IEEE, 5-11 July 2008, pp. 1-4.

[3] M. Lienard, P. Degauque, J. Baudet, and D. Degardin, "Investigation on MIMO channels in subway tunnels," Selected Areas in Communications, IEEE Journal on, vol. 21, no. 3, pp. 332-339, 2003.

[4] J. Molina-Garcia-Pardo, M. Lienard, A. Nasr, and P. Degauque, "On the possibility of interpreting field variations and polarization in arched tunnels using a model for propagation in rectangular or circular tunnels," Antennas and Propagation, IEEE Transactions on, vol. 56, no. 4, pp. 1206-1211, April 2008. 